\title{
REFLECTION OF WAVES IN INITIALLY STRESSED TRANSVERSELY ISOTROPIC FIBRE-REINFORCED THERMOELASTIC MEDIUM
}

\author{
R.R. GUPTA* \\ Department of Mathematics, M.M. University \\ Mullana, Ambala, Haryana, INDIA-133203 \\ E-mail: raji.mmec@gmail.com \\ R.R. GUPTA \\ Department of Mathematics and Applied Sciences \\ MEC, Oman, INDIA \\ E-mail:dr.rajanigupta@gmail.com
}

\begin{abstract}
The present investigation deals with the reflection of plane periodic waves incident at the surface of a homogeneous initially stressed transversely isotropic fibrereinforced thermoelastic medium. The wave equations are solved by imposing proper conditions on displacements, stresses and temperature distribution. Numerically simulated results have been depicted graphically for different angles of incidence with respect to frequency. Some special cases of interest have also been deduced from the present investigation.
\end{abstract}

Key words: reflection, initially stressed, fiber-reinforced, transversely isotropic.

\section{Introduction}

The influence of pre-existing stress on elasticity of solids referred as initial stress and strain or external stress is quite an important subject and has been investigated by a number of researchers. The propagation of elastic waves of a fiber-reinforced medium plays a great role in the practical problems of civil engineering and geophysics. The effects of earthquakes on artificial structures near the surface of the earth is also of prime importance. A structure is excited during an earthquake and similar disturbances, which may cause more or less violent vibrations. These vibrations depend on the ground vibration as well as on the physical properties of the structures (Richter, 1958). Most concrete constructions on or near the surface of the earth include steel reinforcing. The characteristic property of a reinforced concrete member is that its components, namely concrete and steel, act together as a single anisotropic unit as long as they remain in the elastic condition, i.e., the components are bound together without relative displacement. However, due to the mismatch of material properties, there exists a residual stress during the manufacture process of fiberreinforced material. On the contrary, to prevent the fiber-reinforced material from brittel fracture, the layered structure is usually pre-stressed during the manufacture process. During the last five decades considerable attention has been directed towards this phenomenon. Biot (1965) in his work depicted the difference between the acoustic propagation under initial stress and in stress free state.

Acharya and Roy (2008) discussed the propagation of plane waves and their reflection at the free/rigid boundary of a fiber-reinforced magnetoelastic semispace. The influence of rotation, magnetic field, initial stress and gravity on Rayleigh waves in a homogeneous orthotropic elastic medium was investigated by Abd- Alla et al. (2010). However, to the author's knowledge, no work has been carried out so far to discuss the reflection of waves in an initially stressed transversely isotropic fiber-reinforced thermoelastic

\footnotetext{
* To whom correspondence should be addressed
} 
half space. In this article, we will analytically investigate the reflection of waves in an initially stressed transversely isotropic fibre-reinforced thermoelastic medium. The propagation of waves in such materials has many application in various fields of science and technology, namely, atomic physics, industrial engineering, thermal power plants, submarine structures, pressure vessel, aerospace, chemical pipes and metallurgy. A graphical representation is given for amplitude ratios of various reflected waves for different incident waves at different angles of incidence, i.e., for $\theta=30^{\circ}, 45^{\circ}$.

\section{Basic equations}

The linear equations governing thermoelastic interactions in a homogeneous transversely isotropic initially stressed fibre-reinforced thermoelastic solid are

Constitutive relations Following Belfield et al. (1983)

$$
\begin{aligned}
& t_{i j}=\lambda e_{k k} \delta_{i j}+2 \mu_{T} e_{i j}+\alpha\left(a_{k} a_{m} e_{k m} \delta_{i j}+e_{k k} a_{i} a_{j}\right)+ \\
& +2\left(\mu_{L}-\mu_{T}\right)\left(a_{i} a_{k} e_{k j}+a_{j} a_{k} e_{k i}\right)+\beta\left(a_{k} a_{m} e_{k m} a_{i} a_{j}\right)-\beta_{i j} T .
\end{aligned}
$$

The deformation tensor is defined by

$$
e_{i j}=\frac{1}{2}\left(u_{i, j}+u_{j, i}\right), \quad i, j=1,2,3
$$

Balance law: The balance laws for initially stressed fiber-reinforced linearly elastic medium whose preferred direction is that of $\boldsymbol{a}$ are (Spencer, 1972; Dhaliwal and Sherief, 1980; Montanaro, 1999)

$$
t_{i j, j}-P \omega_{i j, j}=\rho \ddot{u}_{i} .
$$

Equation of heat conduction: Following Lord and Shulman (1967)

$$
K_{i j} T_{, i j}=\left(\frac{\partial}{\partial t}+\tau_{0} \frac{\partial^{2}}{\partial t^{2}}\right)\left(T_{0} \beta_{i j} \dot{u}_{i, j}+\rho C_{e} \dot{T}\right), \quad i, j=1,2,3
$$

where $\rho$ is the mass density, $t_{i j}$ are components of stress, $u_{i}$ the mechanical displacement, $e_{i j}$ are components of infinitesimal strain, $P=t_{11}$ is the normal initial stress, $\omega_{i j}=\left(u_{j, i}-u_{i, j}\right) / 2, T$ the temperature change of a material particle, $T_{0}$ the reference uniform temperature of the body, $K_{i j}$ the heat conduction tensor, $\beta_{i j}$ the thermal elastic coupling tensor, $c^{*}$ the specific heat at constant strain, $a_{j}$ are components of $\boldsymbol{a}$, all referred to Cartesian coordinates. The vector $\boldsymbol{a}$ may be a function of position. The coefficients $\lambda, \mu_{L}, \mu_{T}, \alpha$ and $\beta$ are elastic constants with the dimension of stress. We choose $\boldsymbol{a}$ (Belfield et al., 1983) so that its components are $(1,0,0)$. The comma notation is used for spatial derivatives and a superimposed dot represents time differentiation. 


\section{Problem formulation}

Following Slaughter (2002) appropriate transformations have been used on the set of Eq.(2.1), for deriving the equations for a transversely isotropic medium our analysis is restricted to the two dimensional problem.

In the present paper, we take the origin of the coordinate system $\left(x_{1}, x_{2}, x_{3}\right)$ at the free surface of the half space. The $x_{1}-x_{2}$ plane is chosen to coincide with the free surface and $x_{3}$ axis pointing normally into the half-space, which is thus represented by $x_{3} \geq 0$. We consider plane waves in plane such that all particles on a line parallel to the $x_{2}$-axis are equally displaced. Therefore, all the field quantities will be independent of $x_{2}$ coordinate. So, we assume the components of the displacement vector of the form

$$
\boldsymbol{u}=\left(u_{1}, u_{2}, 0\right)
$$

and assume that the solutions are explicitly independent of $x_{3}$, i.e., $\partial / \partial x_{3} \equiv 0$. Thus the field equations and constitutive relations for such a medium reduces to

$$
\begin{aligned}
& c_{11} \frac{\partial^{2} u_{1}}{\partial x_{1}^{2}}+c_{55} \frac{\partial^{2} u_{1}}{\partial x_{2}^{2}}+\left(c_{12}+c_{55}\right) \frac{\partial^{2} u_{3}}{\partial x_{1} \partial x_{2}}-\frac{P}{2}\left(\frac{\partial^{2} u_{2}}{\partial x_{1} \partial x_{2}}-\frac{\partial^{2} u_{1}}{\partial x_{2}^{2}}\right)-\beta_{1} \frac{\partial T}{\partial x_{1}}=\rho \frac{\partial^{2} u_{1}}{\partial t^{2}} \\
& c_{55} \frac{\partial^{2} u_{2}}{\partial x_{1}^{2}}+c_{22} \frac{\partial^{2} u_{2}}{\partial x_{2}^{2}}+\left(c_{12}+c_{55}\right) \frac{\partial^{2} u_{1}}{\partial x_{1} \partial x_{2}}-\frac{P}{2}\left(\frac{\partial^{2} u_{1}}{\partial x_{1} \partial x_{2}}-\frac{\partial^{2} u_{2}}{\partial x_{2}^{2}}\right)-\beta_{2} \frac{\partial T}{\partial x_{1}}=\rho \frac{\partial^{2} u_{2}}{\partial t^{2}} \\
& K_{1} \frac{\partial^{2} T}{\partial x_{1}^{2}}+K_{2} \frac{\partial^{2} T}{\partial x_{2}^{2}}-\rho c^{*}\left(\frac{\partial T}{\partial t}+\tau_{o} \frac{\partial^{2} T}{\partial t^{2}}\right)=T_{o}\left(\frac{\partial}{\partial t}+\tau_{o} \frac{\partial^{2}}{\partial t^{2}}\right)\left(\beta_{1} \frac{\partial u_{1}}{\partial x_{1}}+\beta_{2} \frac{\partial u_{2}}{\partial x_{2}}\right) \\
& t_{22}=c_{12} \frac{\partial u_{1}}{\partial x_{1}}+c_{22} \frac{\partial u_{2}}{\partial x_{2}}-\beta_{2} T, \quad t_{21}=c_{o}\left(\frac{\partial u_{1}}{\partial x_{2}}+\frac{\partial u_{2}}{\partial x_{1}}\right)
\end{aligned}
$$

where $\beta_{1}=\left(c_{11}+c_{13}\right) \alpha_{1}+c_{13} \alpha_{2}, \beta_{2}=\left(c_{33}+c_{13}-c_{55}\right) \alpha_{1}+c_{33} \alpha_{2}, c_{11}=\lambda+2 \alpha+4 \mu_{L}-2 \mu_{T}+\beta, c_{13}=\lambda+\alpha$, $c_{22}=c_{33}=\lambda+2 \mu_{T}, c_{o}=c_{44} / 2, c_{44}=c_{66}=2 \mu_{L}, c_{55}=2 \mu_{T}, c_{23}=c_{33}-c_{55}$, and $\lambda, \alpha, \beta, \mu_{L}, \mu_{T}$ are material constants, $K_{1}, K_{2}$ are coefficients of thermal conductivity, $\tau_{o}$ is thermal relaxation time, $u_{1}, u_{2}$ are the components of the displacement vector.

For further considerations, it is convenient to introduce the non-dimensional quantities defined by

$$
x_{i}^{\prime}=\frac{\omega_{1}^{*} x_{i}}{v_{1}}, \quad u_{i}^{\prime}=\frac{\rho v_{1} \omega_{1}^{*} u_{i}}{\beta_{1} T_{o}}, \quad t_{i j}^{\prime}=\frac{t_{i j}}{\beta_{1} T_{o}}, \quad t^{\prime}=\omega_{1}^{*} t, \quad T^{\prime}=\frac{T}{T_{o}}
$$

where

$$
\omega_{1}^{*}=\frac{c^{*} c_{11}}{k_{1}}, \quad v_{1}^{2}=\frac{c_{11}}{\rho} .
$$




\section{Solution of the problem}

Let $\boldsymbol{p}=\left(p_{1}, p_{2}, 0\right)$ denote the unit propagation vector, $c$ and $k$ are respectively the phase velocity and the wave number of the plane waves propagating in the $x_{1} x_{2}$-plane. We seek a plane wave solution of the equations of motion of the form

$$
\left(u_{1}, u_{2}, T\right)=\left(\bar{u}_{1}, \bar{u}_{2}, \bar{T}\right) e^{i \xi\left(p_{1} x_{1}+p_{2} x_{2}-c t\right)} .
$$

With the help of Eqs (2.9) and (3.1) in Eqs (2.5)-(2.8), we get four homogeneous equations with four unknowns. Solving the resultant system of equations for non-trivial solution, we obtain

$$
A c^{6}+B c^{4}+C c^{2}+D=0
$$

where

$$
\begin{aligned}
& A=g_{6}, \quad B=g_{5}+\omega g_{7}+g_{8} \omega^{-2}, \quad C=\omega g_{4}-g_{2}, \quad D=g_{1}+\omega g_{3}, \\
& a_{i}=\left(d_{i} p_{1}^{2}+p_{3}^{2}\right), i=1,, 5,10, \quad g_{1}=\left(a_{5} a_{1}-d_{2} d_{6} p_{1}^{2} p_{3}^{2}\right) a_{10}, \\
& g_{2}=a_{1}\left[a_{10}\left(d_{8}+d_{9}\right)-p_{3}^{2} d_{7} d_{14}\right]+d_{4} a_{5} a_{10}+ \\
& -p_{1}^{2} p_{3}^{2} d_{2}\left(d_{9} d_{6}+d_{7} d_{13}\right)+p_{1}^{2} d_{3}\left(p_{1}^{2} d_{5} d_{13}+p_{3}^{2}\left(d_{13}+d_{6} d_{14}\right)\right), \\
& g_{3}=-i\left[a_{5}\left(p_{1}^{4} d_{1} d_{11}+p_{3}^{2} a_{1} d_{12}\right)\right]+ \\
& +p_{1}^{2} p_{3}^{2}\left(d_{11}\left(p_{1}^{2}+p_{3}^{2}-d_{2} d_{6} p_{1}^{2}\right)-d_{2} d_{6} d_{12} p_{3}^{2}\right), \\
& g_{4}=-i\left[p_{1}^{2} d_{11}\left(d_{8} a_{1}+d_{4} a_{5}\right)+p_{3}^{2} d_{12}\left(d_{4} a_{5}+d_{8} a_{4}\right)\right], \\
& g_{7}=-i d_{4} d_{8}\left(p_{3}^{2} d_{12}+p_{1}^{2} d_{11}\right), \\
& g_{5}=d_{4} d_{9} a_{10}+p_{1}^{2} d_{8}\left(d_{1} d_{9}+d_{3} d_{13}\right)+p_{3}^{2}\left[d_{8}\left(d_{4}-d_{9}\right)-d_{4} d_{7} d_{14}\right], \\
& g_{6}=d_{4} d_{8} d_{9}, g_{8}=p_{1}^{2} d_{4} d_{8} d_{10} .
\end{aligned}
$$

The roots of this equation give three values of $c 2$. Three positive values of $c$ will be the velocities of propagation of three possible waves. The waves with velocities $c_{1}, c_{2}$, and $c_{3}$ correspond to three types of quasi waves. Let us name these waves as quasi-longitudinal displacement (qLD) wave, quasi transverse displacement wave (qTD) and quasi thermal (qT) wave. 


\section{Reflection of waves}

We consider a homogeneous initially stressed transversely isotropic fibre-reinforced thermoelastic half-space occupying the region $x_{2}>0$. The incident qLD or qT or qTD wave at the interface will generate reflected $\mathrm{qLD}$, qT and $\mathrm{qTD}$ waves in the half space $x_{2}>0$. The total displacements and temperature distribution are given by

$$
\left(u_{1}, u_{2}, T\right)=\sum_{j=1}^{6} A_{j}\left(1, r_{j}, s_{j}\right) e^{i B_{j}}
$$

where

$$
B_{j}=\left\{\begin{array}{l}
\omega\left(t-x_{1} \sin e_{j}-x_{2} \cos e_{j}\right) / c_{j}, j=1,2,3, \\
\omega\left(t-x_{1} \sin e_{j}+x_{2} \cos e_{j}\right) / c_{j}, j=4,5,6,
\end{array}\right.
$$

$\omega$ is the angular frequency. Here subscripts $1,2,3$ respectively denote the quantities corresponding to the incident qLD, qT and qTD wave whereas the subscripts 4,5 and 6 respectively denote the corresponding reflected waves and

$$
\begin{aligned}
& r_{j}=\frac{\Delta_{1 j}}{\Delta_{j}}, \quad s_{j}=\frac{\Delta_{2 j}}{\Delta_{j}}, \quad j=1,2,3, \\
& \Delta_{j}=\left|\begin{array}{cc}
p_{2}^{2}+p_{1}^{2}\left(d_{6}-d_{8}\right)-c_{i}^{2} d_{9} & i p_{2} d_{7} \\
i p_{2} \bar{\beta} k \varepsilon_{2} c_{j}\left(i+\tau_{o} \omega\right) & -k^{2}\left(p_{1}^{2}+p_{2}^{2} \bar{k}\right)+k c_{j} \varepsilon_{l}\left(i+\tau_{o} \omega\right)
\end{array}\right|, \\
& \Delta_{1 j}=\left|\begin{array}{cc}
p_{1} p_{2}\left(p_{1} d_{5}+d_{6}\right) & i p_{2} d_{7} \\
p_{1} k \varepsilon_{2} c_{i}\left(i+\tau_{o} \omega\right) & -k^{2}\left(p_{1}^{2}+p_{2}^{2} \bar{k}\right)+k c_{j} \varepsilon_{1}\left(i+\tau_{o} \omega\right)
\end{array}\right|, \\
& \Delta_{2 j}=\left|\begin{array}{cc}
p_{1} p_{2}\left(p_{1} d_{5}+d_{6}\right) & p_{2}^{2}+p_{1}^{2}\left(d_{6}-d_{8}\right)-c_{i}^{2} d_{9} \\
p_{1} k \varepsilon_{2} c_{i}\left(i+\tau_{o} \omega\right) & p_{2} \bar{\beta} k \varepsilon_{2} c_{j}\left(i+\tau_{o} \omega\right)
\end{array}\right| .
\end{aligned}
$$

For the incident qLD-wave: $p_{1}=\sin e_{1}, p_{2}=\cos e_{1}$, qT-wave: $p_{1}=\sin e_{2}, p_{2}=\cos e_{2}$, qTD-wave: $p_{1}=\sin e_{3}, p_{2}=\cos e_{3}$, For the reflected qLD-wave: $p_{1}=\sin e_{4}, p_{2}=\cos e_{4}$, ; qT-wave: $p_{1}=\sin e_{5}, p_{2}=\cos e_{5}$, qTD-wave: $p_{1}=\sin e_{6}, p_{2}=\cos e_{6}$.

Here $e_{1}=e_{4}, e_{2}=e_{5}$ and $e_{3}=e_{6}$, i.e., the angle of incidence is equal to the angle of reflection in the generalized thermoelastic transversely isotropic medium, so that the velocities of reflected waves are equal to their corresponding to incident waves i.e., $c_{1}=c_{4}, c_{2}=c_{5}$ and $c_{3}=c_{6}$. 


\section{Boundary conditions}

The boundary conditions are given by

$$
t_{22}=0, \quad t_{21}=0, \quad \frac{\partial T}{\partial x_{2}}+h T=0
$$

where $h$ is the surface heat transfer coefficient;

$h \rightarrow 0$ corresponds to thermally insulated boundaries and

$h \rightarrow \infty$ refers to isothermal boundaries.

$$
t_{22}=\frac{C_{12}}{C_{22}} \frac{\partial u_{1}}{\partial x_{1}}+\frac{\partial u_{2}}{\partial x_{2}}-\frac{\beta_{22}}{C_{12}} T, \quad t_{21}=\frac{C_{55}}{C_{22}}\left(\frac{\partial u_{1}}{\partial x_{2}}+\frac{\partial u_{2}}{\partial x_{1}}\right)
$$

The wave numbers $\xi_{j}, j=1,2, \ldots .6$ and the apparent velocity $c_{j}, j=1,2, \ldots .6$ are connected by the relation

$$
c_{1} \xi_{1}=c_{2} \xi_{2}=\ldots \ldots \ldots . . .=c_{6} \xi_{6}=\omega
$$

at the surface $x_{3}=0$. Relation (6.2) may also be written in order to satisfy the boundary conditions (6.1) as

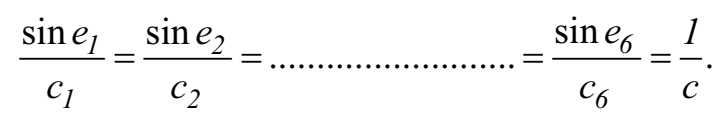

Making use of Eqs (3.4), (4.1), (6.2) and (6.3) into thermally insulated boundary conditions (6.1), we obtain

$$
\sum_{j=1}^{6} A_{i j} A_{j}=0, \quad i=1,2,3
$$

where

$$
\begin{gathered}
A_{1 j}=\left\{\begin{array}{l}
\frac{C_{12}}{C_{11}} \frac{\sin e_{j}}{c_{j}}+r_{j} \frac{\cos e_{j}}{c_{j}}-\frac{\beta_{2} s_{j}}{C_{11}}, j=1,2,3, \\
\frac{C_{12}}{C_{11}} \frac{\sin e_{j}}{c_{j}}-r_{j} \frac{\cos e_{j}}{c_{j}}-\frac{\beta_{2} s_{j}}{C_{11}}, j=4,5,6,
\end{array}\right. \\
A_{2 j}=\left\{\begin{array}{l}
\frac{C_{55}}{C_{11}}\left[\frac{\cos e_{j}}{c_{j}}-r_{j} \frac{\sin e_{j}}{c_{j}}, j=1,2,3,\right. \\
\frac{C_{55}}{C_{11}}\left[\frac{\cos e_{j}}{c_{j}}+r_{j} \frac{\sin e_{j}}{c_{j}}, j=4,5,6,\right.
\end{array}\right.
\end{gathered}
$$




$$
A_{3 j}=\left\{\begin{aligned}
s_{j} \frac{\cos e_{j}}{c_{j}}, j=1,2,3 \\
-s_{j} \frac{\cos e_{j}}{c_{j}}, j=4,5,6
\end{aligned}\right.
$$

\section{Incident qLD-wave}

In the case of incident qLD- wave, $A_{2}=A_{3}=0$. Dividing the set of Eq.(6.5) throughout by $A_{1}$, we obtain a system of three non-homogeneous equations with three unknowns which can be solved by Gauss elimination method and we have

$$
Z_{i}=\frac{A_{i+3}}{A_{l}}=\frac{\Delta_{i}^{l}}{\Delta}, \quad i=1,2,3 .
$$

\section{Incident qT-wave}

In the case of the incident qT- wave, $A_{1}=A_{2}=0$ and thus we have

$$
Z_{i}=\frac{A_{i+3}}{A_{2}}=\frac{\Delta_{i}^{2}}{\Delta}, \quad i=1,2,3 .
$$

\section{Incident qTD-wave}

In the case of the incident qTD- wave, $A_{1}=A_{2}=0$ and thus we have

$$
Z_{i}=\frac{A_{i+3}}{A_{3}}=\frac{\Delta_{i}^{3}}{\Delta}, \quad i=1,2,3
$$

where $\Delta=\left|A_{i i+3}\right|_{3 \times 3}$ and $\Delta_{i}^{p}(i=1,2,3, p=1,2,3)$ can be obtained by replacing, respectively, the $1^{\text {st }}, 2^{\text {nd }}$, $3^{\text {rd }}$ column of $\Delta$ by $\left[-A_{1 p}-A_{2 p}-A_{3 p}\right]^{T}$.

\section{Numerical results and discussion}

In order to illustrate the theoretical results obtained in the preceding sections, we now present some numerical results. For the purpose of numerical computations, we have used Matlab's Programming. The following relevant physical constants are taken (Singh, 2006) for a fiber-reinforced transversely isotropic material,

$$
\begin{aligned}
& \rho=2.66 \times 10^{3} \mathrm{Kg} / \mathrm{m}^{3}, \quad \lambda=5.65 \times 10^{10} \mathrm{~N} / \mathrm{m}^{2}, \quad \mu_{T}=2.46 \times 10^{10} \mathrm{~N} / \mathrm{m}^{2}, \quad \mu_{L}=5.66 \times 10^{10} \mathrm{~N} / \mathrm{m}^{2}, \\
& \alpha=-1.28 \times 10^{10} \mathrm{~N} / \mathrm{m}^{2}, \quad \beta=220.90 \times 10^{10} \mathrm{~N} / \mathrm{m}^{2}, \quad K_{l}=.0921 \times 10^{3} \mathrm{Jm}^{-1} \mathrm{deg}^{-1} \mathrm{~s}^{-1},
\end{aligned}
$$




$$
\begin{aligned}
& K_{2}=.0963 \times 10^{3} \mathrm{Jm}^{-1} \mathrm{deg}^{-1} \mathrm{~s}^{-1}, \quad \alpha_{1}=.017 \times 10^{4} \mathrm{deg}^{-1}, \quad \alpha_{2}=.015 \times 10^{4} \mathrm{deg}^{-1}, \\
& c^{*}=0.787 \times 10^{3} \mathrm{~J} \mathrm{Kg}^{-1} \mathrm{deg}^{-1}, \quad T_{0}=293 \mathrm{~K}, \quad P=100, \quad \tau_{0}=.05 \mathrm{~s}, \quad \omega=2 \mathrm{~s}^{-1} .
\end{aligned}
$$

A graphical representation is given for the variations of amplitude ratios of reflected qLD, qTD and qT waves when three types of waves, viz., qLD, qTD and qT waves are incident at the free surface to compare the results in two cases, one for the waves incident from initially stressed fiber- reinforced thermoelastic transversely isotropic (ISFRTIT) and other from initially stressed fiber-reinforced thermoelastic isotropic (ISFRIT). In Figs 1-3, a graphical representation is given for variations of amplitude ratios $|Z 1|,|Z 2|$ and $|Z 3|$ in the case of the incident qLD wave. Figures 4-6 and 7-9, respectively show the same cases when qTD and qT waves are incident. Here $|Z 1|,|Z 2|$ and $|Z 3|$ are, respectively, the amplitude ratios of reflected qLD, qTD and qT waves. These variations are shown for two angles of incidence, viz., $\theta=30^{\circ}$, $45^{\circ}$. In these figures the solid curves correspond to the case of ISFRTIT, while broken curves correspond to the case of ISFRIT. Also, the curves without a center symbol correspond to the case, when $\theta=30^{\circ}$ and curves with a center symbol $(-o-$ $o^{-}$) represent the variation corresponding to the case of $\theta=45^{\circ}$.

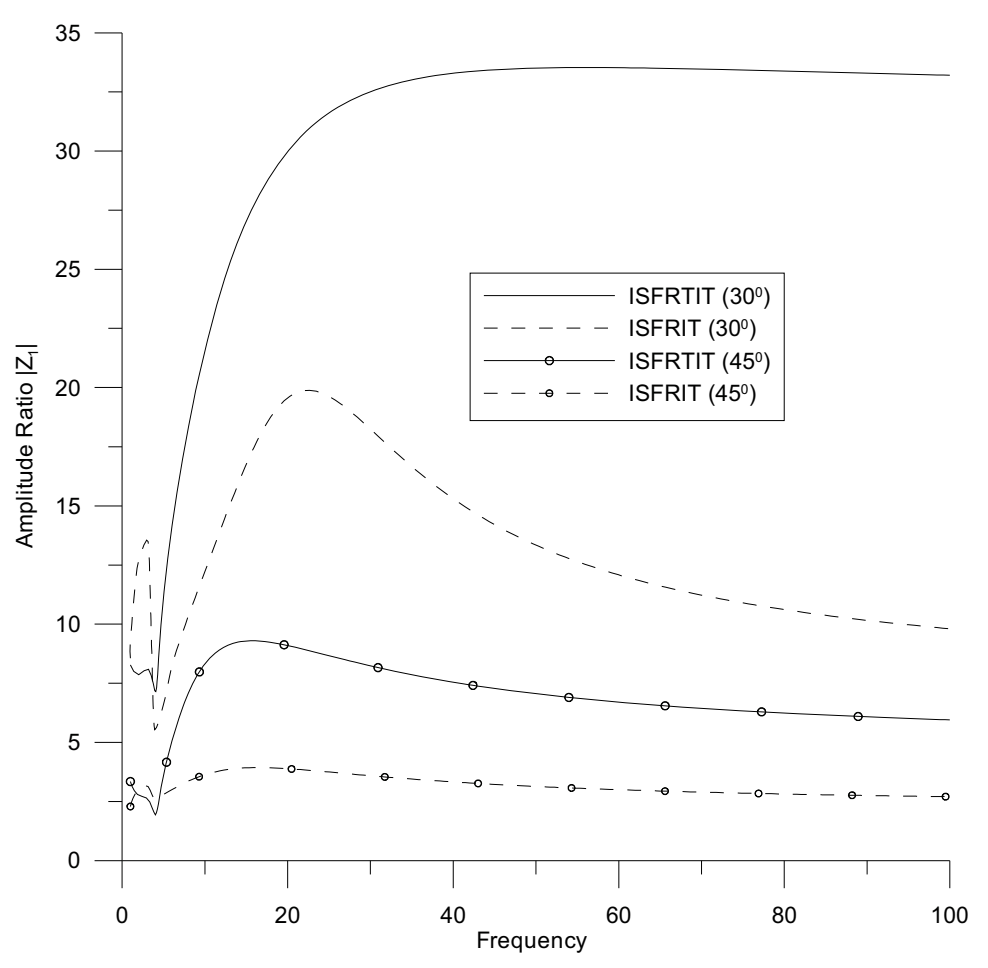

Fig.1. Amplitude ratio $\left|Z_{l}\right|$ when qLT wave is incident. 


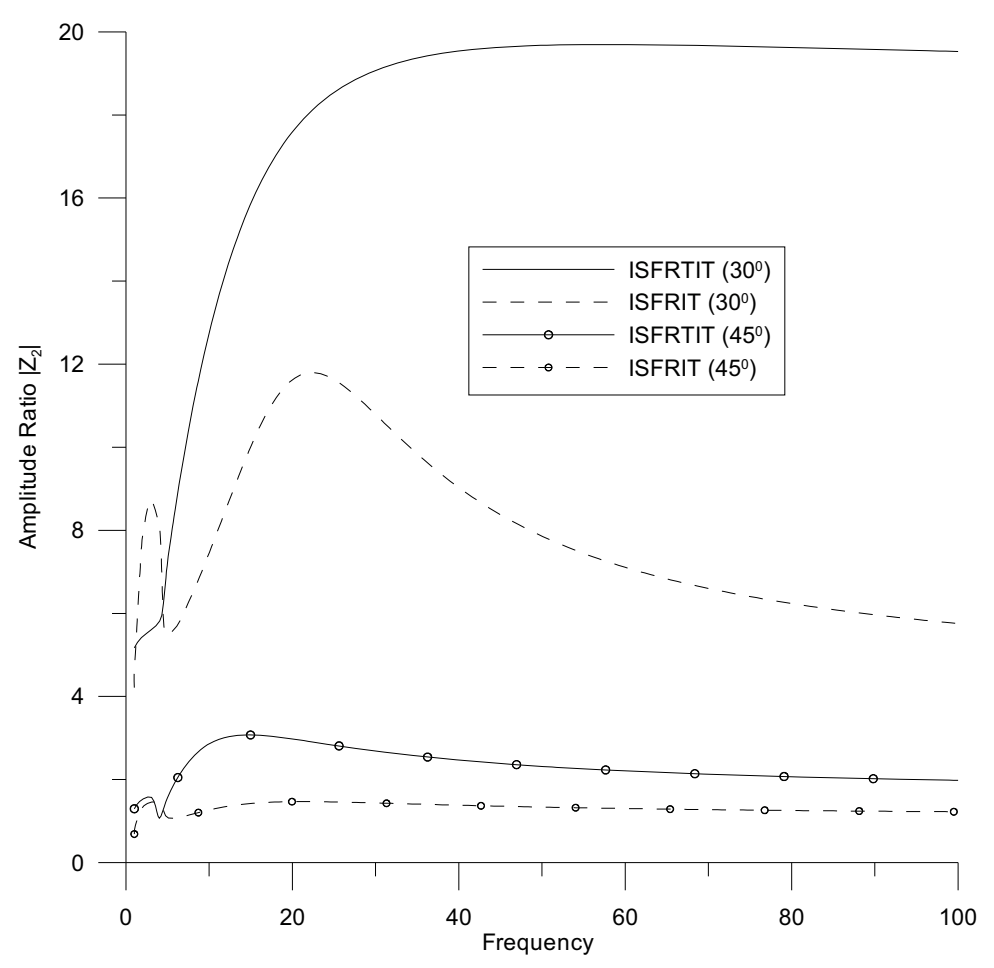

Fig.2. Amplitude ratio $\left|Z_{2}\right|$ when $\mathrm{qLT}$ wave is incident.

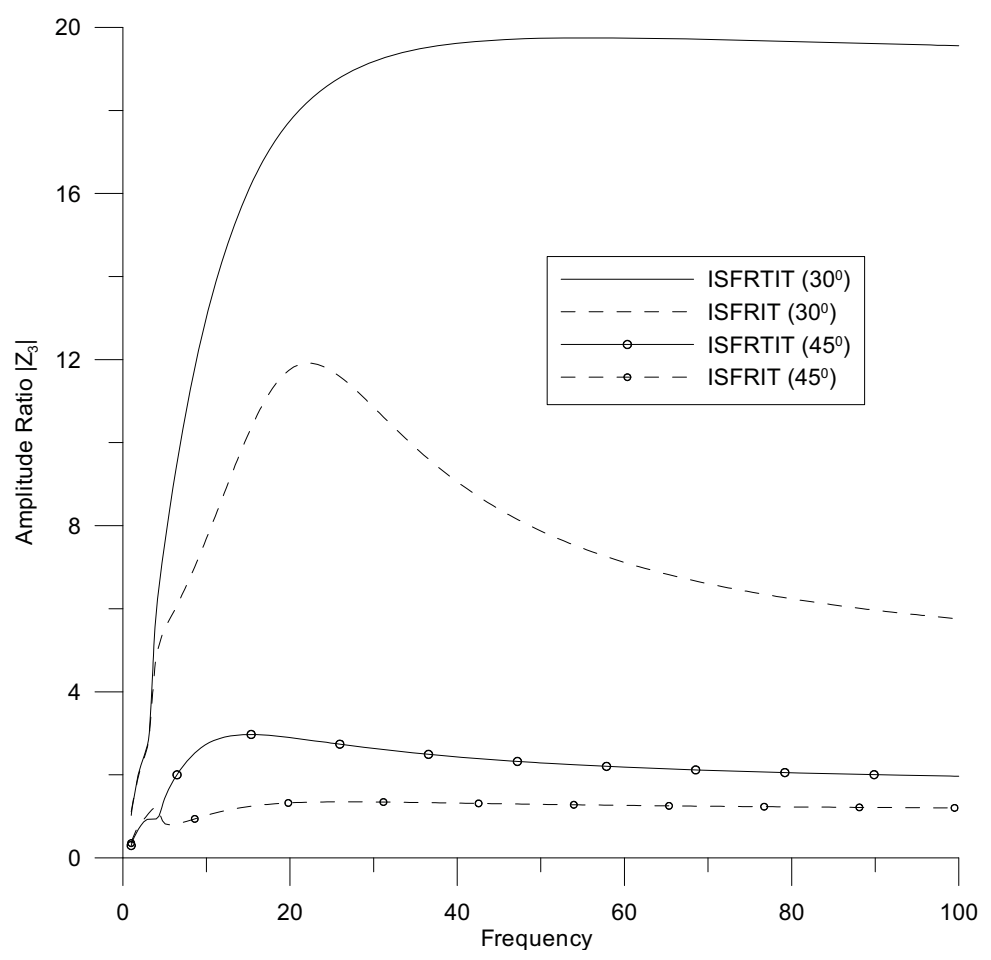

Fig.3. Amplitude ratio $\left|Z_{3}\right|$ when qLT wave is incident. 


\section{Incident qLD - wave}

It is evident from Figs 1 and 2 that the amplitude ratios $\left|Z_{1}\right|$ and $\left|Z_{2}\right|$ of the reflected qLD and qTD wave, for $\theta=30^{\circ}$ and for both ISFRTIT and ISFRIT, first oscillate within the interval $(0,2)$, then sharply increase to attain a maximum value up to $\omega=20$, then become constant for ISFRTIT and decrease to become constant for ISFRIT. Similar variations are depicted for $\theta=45^{\circ}$ but with a difference in their amplitudes. Figure 3 indicates the variation of the amplitude ratio $\left|Z_{3}\right|$ of reflected qT-waves, which shows that for the case of ISFRTIT and ISFRIT, its value shows the similar variations as those depicted in the case of $\left|Z_{l}\right|$, with difference in their initial region of oscillation. In the present case for $\theta=30^{\circ}$ its value strictly increases with an increase in frequency up to $\omega=20$. The variations are almost similar for $\theta=45^{\circ}$ except with difference in their amplitudes.

\section{Incident qTD - wave}

The variation in the amplitude ratio of the reflected wave for incident qTD wave is shown in Figs 46. It is follows from these figures that the value of amplitude ratio of $\left|Z_{I}\right|$ starts with initial oscillation within the interval $(0,10)$ and then becomes constant.

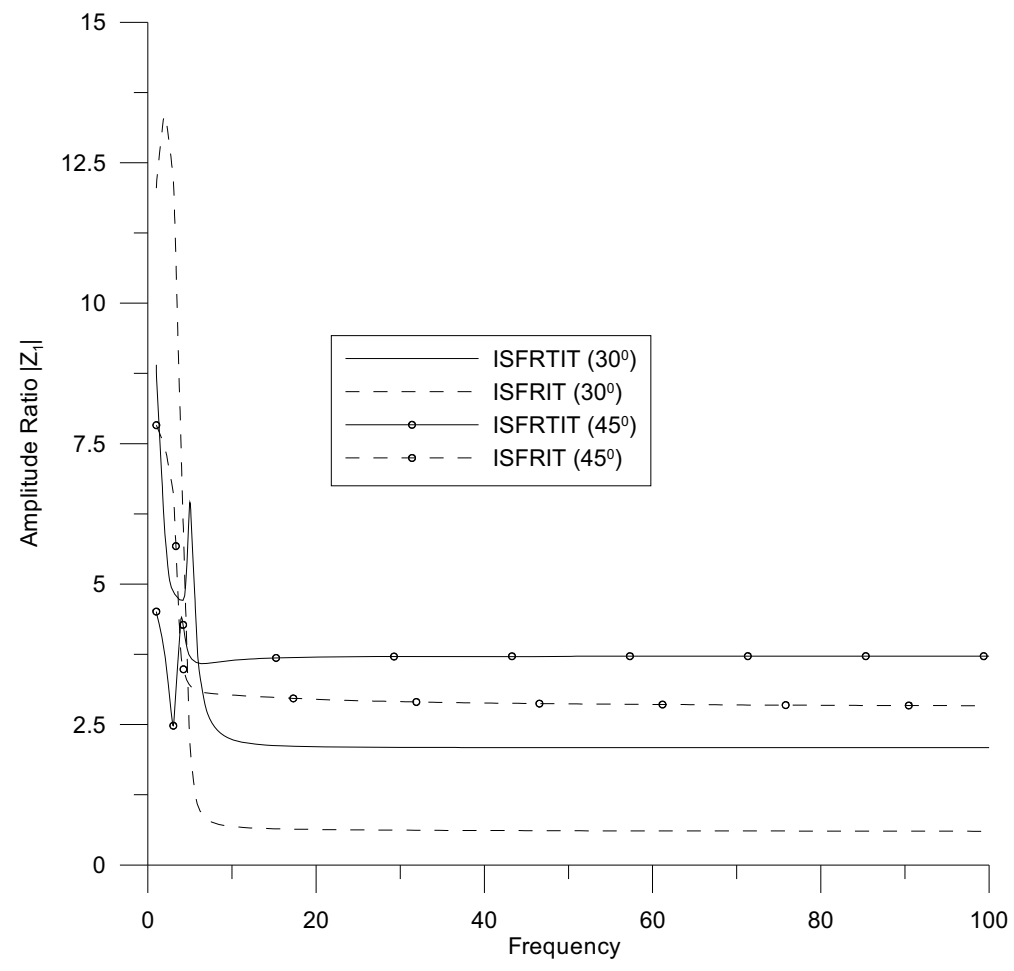

Fig.4. Amplitude ratio $\left|Z_{l}\right|$ when qTD wave is incident. 


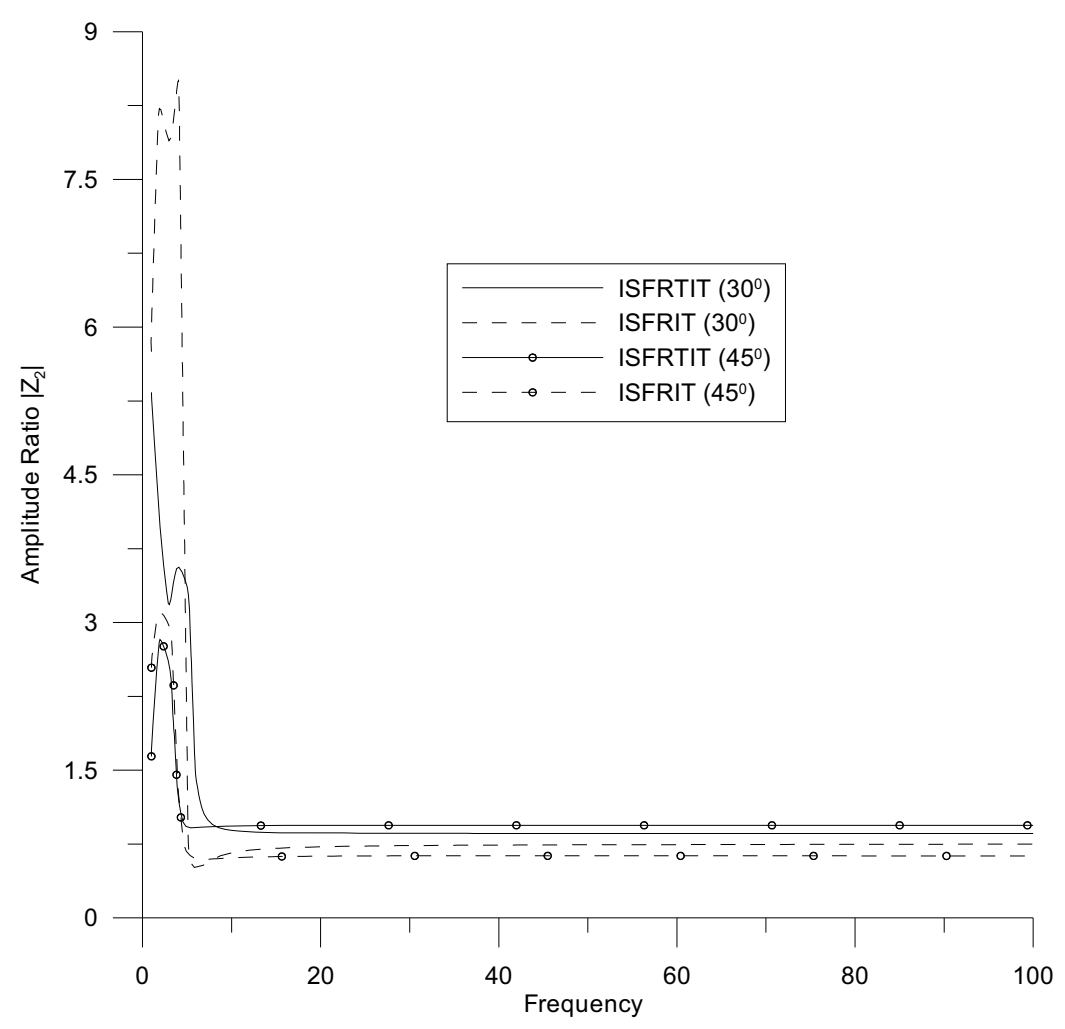

Fig.5. Amplitude ratio $\left|Z_{2}\right|$ when qTD wave is incident.

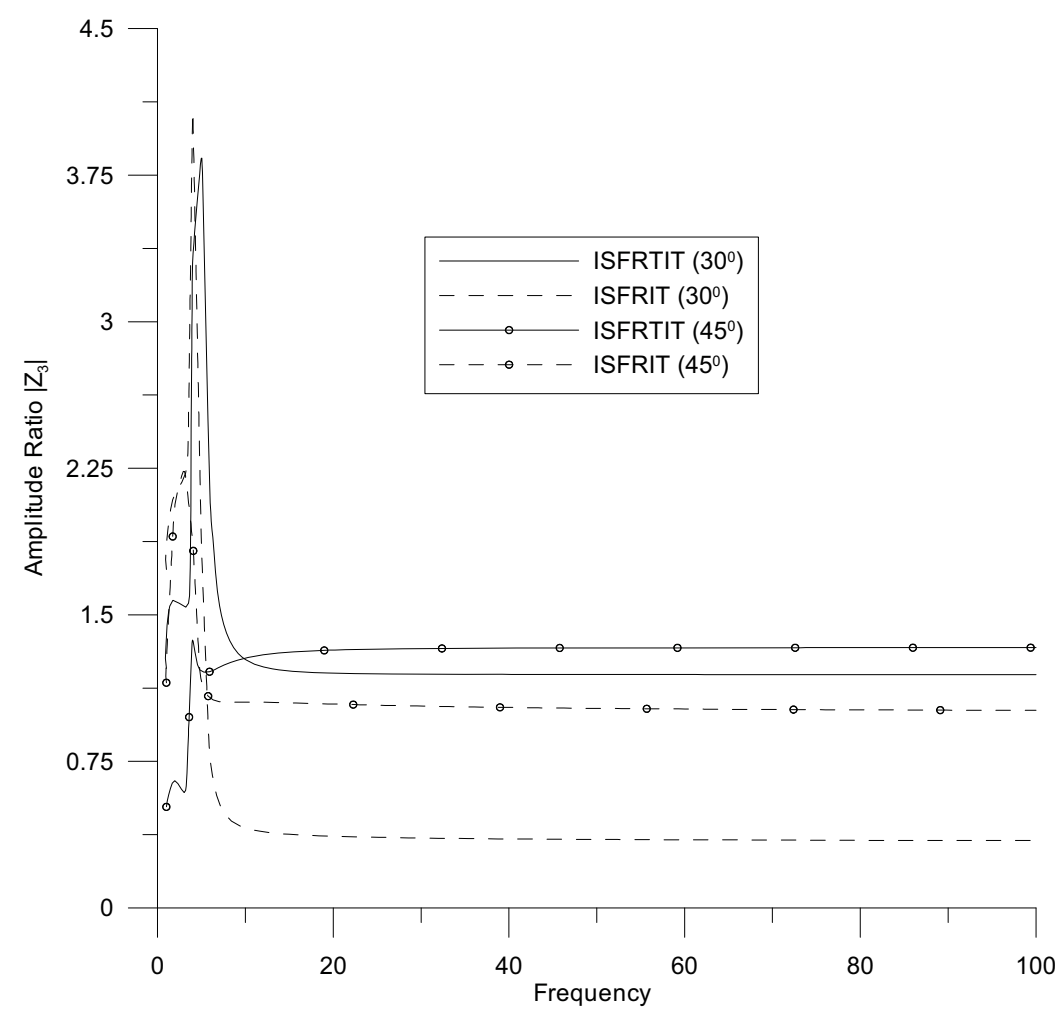

Fig.6. Amplitude ratio $\left|Z_{3}\right|$ when qTD wave is incident. 


\section{Incident $q T$ - wave}

Figures 7-9 illustrates the variations of amplitude ratios of $Z i, i=1,2,3$, with frequency. It can be seen from these figures that the variation pattern of the amplitudes is almost similar with a difference in their peak values. Their values show a hump within an interval and after that they tend to attain a constant value. The amplitude ratio of the first wave gets increased due to anisotropy, while for the remaining two waves, its value gets decreased. It is also seen from the graphs that at $\theta=30^{\circ}$ and ISFRTIT the values of amplitude ratios of $Z i, i=1,2,3$, initially oscillate with small amplitude and then flatten to become constant. The value of amplitude ratio $Z_{l}$ becomes increased with an increase in the angle $\theta$ for both the cases of ISFRTIT and ISFRIT.

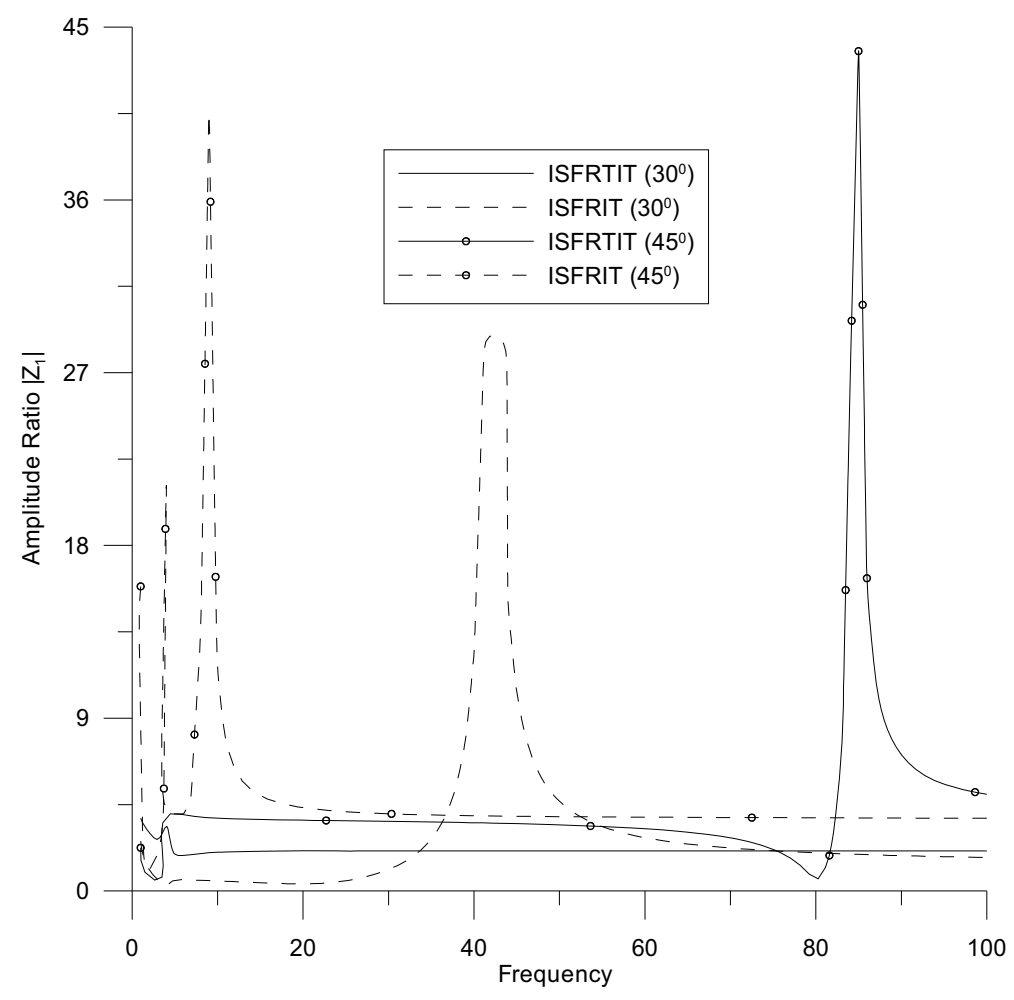

Fig.7. Amplitude ratio $\left|Z_{l}\right|$ when qT wave is incident. 


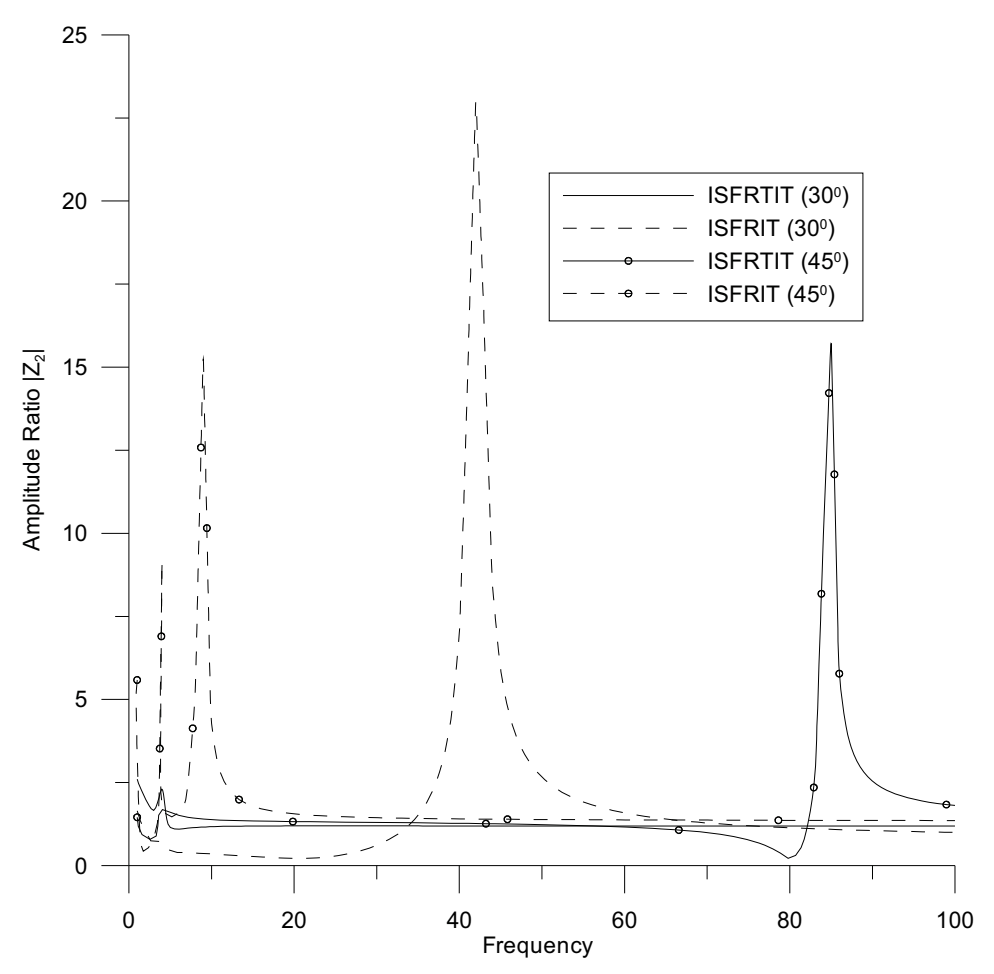

Fig.8. Amplitude ratio $\left|Z_{2}\right|$ when qT wave is incident.

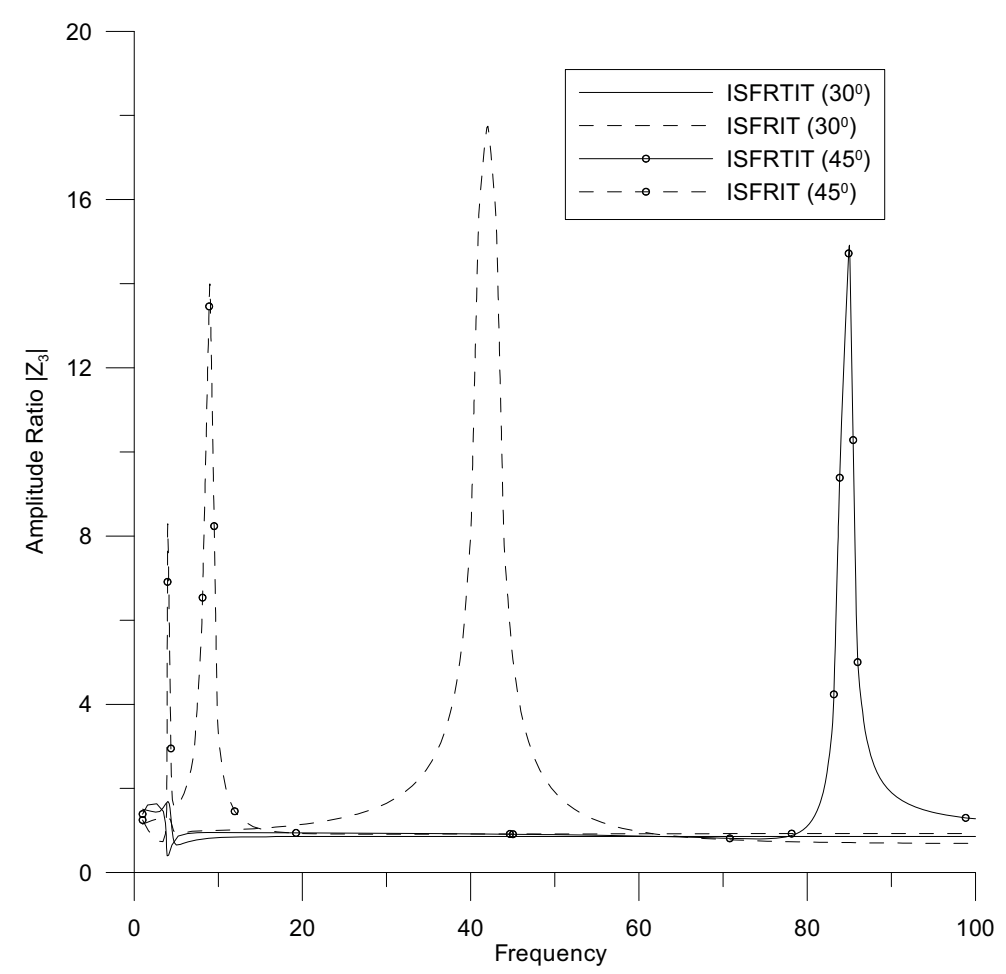

Fig.9. Amplitude ratio $\left|Z_{3}\right|$ when qT wave is incident. 


\section{Conclusion}

The importance of thermal stresses in causing structural damages and changes in functioning of the structure is well recognized whenever thermal stress environments are involved. Plane wave reflection from the free surface of initially stressed transversely isotropic fiber-reinforced thermoelastic medium has been discussed. An appreciable effect of anisotropy and angle of incidence is observed on amplitude ratios of various reflected waves. It is concluded from the graphs that the values of amplitude ratios $\left|Z_{1}\right|$ and $\left|Z_{2}\right|$ get decreased due to an increase in anisotropy for both the angle of oscillation, while the reverse behavior is observed for the amplitude ratio $\left|Z_{3}\right|$.

\section{Nomenclature}

$$
\begin{aligned}
& a_{j} \text { - components of } \boldsymbol{a} \text {, all referred to Cartesian coordinates } \\
& c^{*}-\text { specific heat at constant strain } \\
& e_{i j} \text { - components of infinitesimal strain } \\
& K_{i j} \quad \text { - heat conduction tensor } \\
& P=t_{11} \quad \text { - the normal initial stress } \\
& T_{0} \text { - reference uniform temperature of the body } \\
& t_{i j} \text { - components of stress } \\
& u_{i}-\text { mechanical displacement } \\
& \beta_{i j}-\text { thermal elastic coupling tensor } \\
& \rho \text { - mass density } \\
& \omega_{i j}=\left(u_{j, i}-u_{i, j}\right) / 2, T \text { - temperature change of a material particle }
\end{aligned}
$$

The vector $\boldsymbol{a}$ may be a function of position.

Coefficients $\lambda, \mu_{L}, \mu_{T}, \alpha$ and $\beta$ are elastic constants with the dimension of stress.

$\boldsymbol{a}$ is choosen in a way so that its components are $(1,0,0)$.

The comma notation is used for spatial derivatives and a superimposed dot represents time differentiation.

\section{References}

Abd-Alla A.M., Mahmoud S.R., Abo-Dahab S.M. and Helmy M.I. (2010): Influences of rotation, magnetic field, initial stress and gravity on Rayleigh waves in a homogeneous orthotropic elastic half-space. - Applied Mathematical Sciences, vol.4, pp.91-108.

Acharya D.P. and Roy I. (2008): Propagation of plane waves and their reflection at the free/rigid boundary of a fiberreinforced magnetoelastic semispace. - Int. J. of Appl. Math. and Mech., vol.4, pp.39-58.

Belfield A.J., Rogers T.G. and Spencer A.J.M. (1938): Stress in elastic plates reinforced by fibres lying in concentric circles. - J. Mech. Phys. Solids, vol.31, pp.25-54.

Biot M.A. (1965): Mechanics of Incremental Deformation. - Inc New York: John Wiley and Sons.

Dhaliwal R.S. and Sherief H.H. (1980): Generalized thermoelasticity for anisotropic media. - Q. Appl. Math., vol.33, pp.1-8.

Lord H.W. and Shulman Y. (1967): A generalized dynamical theory of thermoelasticity. - J. Mech. Phys. Solid, vol.15, pp.299-306.

Montanaro A. (1999): On singular surface in isotropic linear thermoelasticity. - J. Acoust. Soc. Am., vol.15, pp.15861588. 
Richter C.F. (1958): Elementary Seismology. - San Francisco, London: W.H. Freeman.

Singh B. (2006): Wave propagation in thermally conducting linear fibre-reinforced. - Composite Materials, vol.75, pp.513-520.

Slaughter W.S. (2002): The linearized theory of elasticity. - Birkhauser.

Spencer A.J.M. (1972): Deformation of Fibre-Reinforced Materials. - London: Oxford University Press.

Received: March 30, 2011

Revised: June 3, 2013 